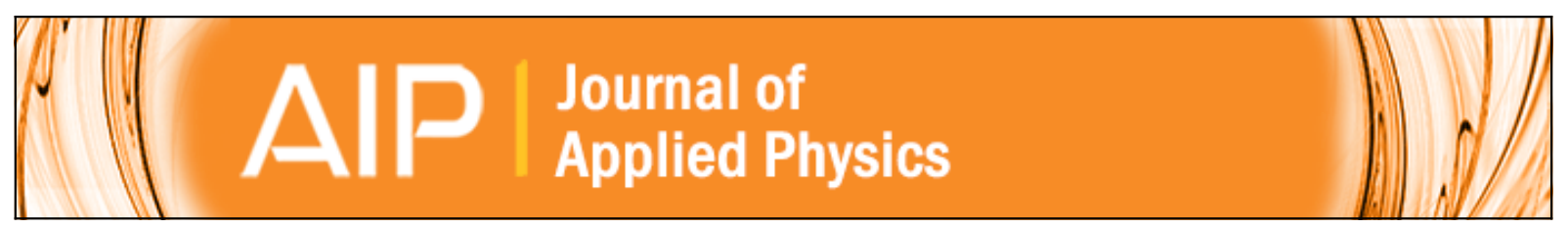

\title{
Milling effects on magnetic properties of melt spun Fe-Nb-B alloy
}

\section{J. J. Ipus, J. S. Blázquez, V. Franco, M. Stoica, and A. Conde}

Citation: Journal of Applied Physics 115, 17B518 (2014); doi: 10.1063/1.4866700

View online: http://dx.doi.org/10.1063/1.4866700

View Table of Contents: http://scitation.aip.org/content/aip/journal/jap/115/17?ver=pdfcov

Published by the AIP Publishing

\section{Articles you may be interested in}

Analysis of heating effects (magnetic hyperthermia) in $\mathrm{FeCrSiBCuNb}$ amorphous and nanocrystalline wires

J. Appl. Phys. 111, 07 A314 (2012); 10.1063/1.3672850

Magnetocaloric response of $\mathrm{Fe} 75 \mathrm{Nb} 10 \mathrm{~B} 15$ powders partially amorphized by ball milling

J. Appl. Phys. 105, 123922 (2009); 10.1063/1.3155982

Effect of Co substitution on the crystallization behavior and magnetic properties of melt-spun ( $\mathrm{Pr}, \mathrm{Tb}$ ) 2 ( $\mathrm{Fe}, \mathrm{Nb}, \mathrm{Zr}$ ) $14 \mathrm{~B} / \mathrm{a}-\mathrm{Fe}$ nanocomposites

J. Appl. Phys. 93, 7978 (2003); 10.1063/1.1558273

Dependence of the magnetic and magnetoelastic properties of ( TbFe 2 ) 1-x B x melt-spun alloys on $\mathrm{x}$ J. Appl. Phys. 85, 4500 (1999); 10.1063/1.370388

Magnetic properties of Nd 8 Fe 77 Co 5 B 6 CuNb 3 melt-spun ribbons

J. Appl. Phys. 83, 6628 (1998); 10.1063/1.367928

\section{SHIMADZU Powerful, Multi-functional UV-Vis-NIR and Excellence in science FTIR Spectrophotometers}

Providing the utmost in sensitivity, accuracy and resolution for applications in materials characterization and nano research

- Photovoltaics - Ceramics

- Polymers

- Thin films

- Paints

- DNA film structures

- Coatings

- Packaging materials
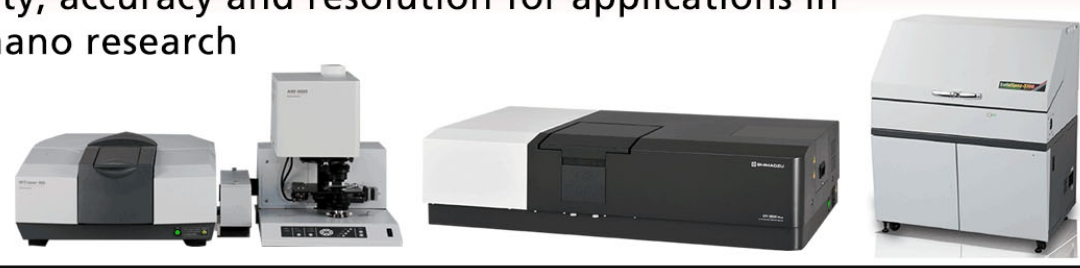


\title{
Milling effects on magnetic properties of melt spun Fe-Nb-B alloy
}

\author{
J. J. Ipus, ${ }^{1, a)}$ J. S. Blázquez, ${ }^{1}$ V. Franco, ${ }^{1}$ M. Stoica, ${ }^{2}$ and A. Conde ${ }^{1}$ \\ ${ }^{1}$ Departamento de Física de la Materia Condensada, ICMSE-CSIC, Universidad de Sevilla, \\ P.O. Box 1065, 41080 Sevilla, Spain \\ ${ }^{2}$ IFW Dresden, Institute for Complex Materials, Helmholtzstr. 20, D-01069 Dresden, Germany
}

(Presented 7 November 2013; received 20 September 2013; accepted 22 November 2013; published online 25 February 2014)

\begin{abstract}
$\mathrm{Fe}_{75} \mathrm{Nb}_{10} \mathrm{~B}_{15}$ amorphous ribbons were grinded via ball milling to produce powder samples preserving the amorphous microstructure. A continuous increase of the Curie temperature with the milling time is observed as well as an enhancement of spontaneous magnetization, average hyperfine field, and magnetocaloric effect. This enhancement in the magnetic character of the samples as milling progresses is ascribed to an increase of the Fe-Fe distance. However, the peak entropy change reduces after grinding the ribbon sample. This effect could be related to a broader distribution of Curie temperatures in powdered samples. C 2014 AIP Publishing LLC.
\end{abstract}

[http://dx.doi.org/10.1063/1.4866700]

\section{INTRODUCTION}

Magnetocaloric effect at room temperature (RT) has deserved an increased attention of the research community in the last years ${ }^{1}$ as the RT magnetic refrigeration is a promising technology, more energetically efficient compared to other types of RT conventional ones. Moreover, this new technology reduces the environmental impact avoiding the use of ozone depleting and global warming substances.

Fe-based amorphous alloys have been proposed to reduce material costs with respect to rare earth based compounds. Moreover, the second order phase transition present in these materials and their soft magnetic character yield negligible thermal and magnetic hysteresis losses. Although the research on magnetocaloric materials for RT applications has risen since the 90's, especially after the discovery of $\mathrm{Gd}_{5} \mathrm{Ge}_{2} \mathrm{Si}_{2}$ compound, ${ }^{2}$ the study of powder materials was delayed till the first decade of present century. ${ }^{3,4}$

In order to produce powder samples, ball milling is a suitable technique that allows us to prepare a large amount of material. The microstructural evolution of the starting samples depends on several factors, such as the energy supplied to the powder during milling. There is a wide variety of transformations that can be induced by ball milling as amorphization, formation of metastable phases (solid solutions, quasicrystals), recrystallization of an amorphous phase, etc. ${ }^{5}$ When the starting system is heterogeneous, homogenization of the powder via mechanical alloying is achieved.

Although B is usually present in soft magnetic amorphous compositions, its atomic dissolution in the matrix via mechanical alloying is not completely successful when pure $\mathrm{B}$ phases are present in the starting mixture. ${ }^{6}$ Even the presence of impurities in $\mathrm{FeB}$ intermetallics leads to remaining $\mathrm{B}$ inclusions, which yield a lower B content in the amorphous phase than the nominal value and thus affecting several properties as crystallization and magnetic ones. ${ }^{6}$

\footnotetext{
a) Author to whom correspondence should be addressed. Electronic mail: jhonipus@us.es.
}

In this work, amorphous $\mathrm{Fe}_{75} \mathrm{Nb}_{10} \mathrm{~B}_{15}$ melt spun ribbons were ball milled in order to study the evolution of the magnetocaloric response of the resulting powder samples as a function of the milling time while the initial amorphous structure is preserved during the process.

\section{EXPERIMENTAL}

$\mathrm{Fe}_{75} \mathrm{Nb}_{10} \mathrm{~B}_{15}$ amorphous alloy ribbon was produced by melt-spinning technique starting from high purity precursors which were arc-melted in Ar atmosphere. $15 \mathrm{~g}$ of ribbon was cut in pieces and introduced into the vials with a ball to powder ratio 10:1. Ball milling was performed in a planetary mill RETSCH PM400 using a rotational speed of $350 \mathrm{rpm}$. After some selected milling times, powder samples were taken out from the vials to characterize their microstructural evolution and magnetic properties.

Amorphous structure of the melt spun sample was checked by X-ray diffraction (XRD) using $\mathrm{Cu}-\mathrm{K} \alpha$ radiation in a Bruker D8I diffractometer. Local Fe environments were analyzed by Mossbauer spectrometry at room temperature in transmission geometry using a ${ }^{57} \mathrm{Co}(\mathrm{Rh})$ source. The values of the hyperfine parameters were obtained by fitting with the different spectra imposing a hyperfine field distribution from 0 to $33 \mathrm{~T}$ in NORMOS program. ${ }^{7}$ A figure with Mössbauer spectra and hyperfine field distributions is available as supplementary material. ${ }^{8}$ Isothermal magnetization curves were measured using a Lakeshore 7407 vibrating sample magnetometer (VSM) with a maximum applied field of $H=1.5 \mathrm{~T}$, field steps of $250 \mathrm{Oe}$ and in the temperature range from $77 \mathrm{~K}$ to $573 \mathrm{~K}$ with increments of $10 \mathrm{~K}$. Spontaneous magnetization, $\sigma_{0}$, was obtained from the extrapolation of high field magnetization curves, $M(H)$, to zero field. Curie temperature, $T_{C}$, was calculated as the inflexion point of $\sigma_{0}(T)$ curves. Magnetic entropy change, $\Delta S_{M}$ due to the application of a magnetic field has been calculated by processing the temperature and field dependent magnetization curves using a numerical approach to the equation 


$$
\Delta S_{M}=\int_{0}^{H_{M A X}}\left(\frac{d M}{d T}\right)_{H} d H .
$$

The partial derivatives are replaced by finite differences and the integration is performed numerically from zero field to the maximum value $H_{M A X}$.

\section{RESULTS AND DISCUSSION}

Microstructural evolution with milling process is shown in the XRD patterns of Figure 1. It can be seen that the amorphous structure of the initial melt spun sample is preserved throughout the explored milling time. In order to extract some information from the patterns, these were fitted using a Gaussian profile for the amorphous phase and imposing a Lorentzian profile to simulate possible nanocrystals in the $2 \theta$ range where the (110) bcc-Fe diffraction line should appear. No important crystalline contribution was found in the patterns (estimated crystalline fraction $<2 \%$, below the precision of the method). The position of the halo shifts to lower $2 \theta$ values as milling progresses, which indicates an expansion of the average Fe-Fe distance. The inset of Figure 1 shows the evolution of the metal-metal distances estimated as ${ }^{9}$

$$
\delta_{M-M}=\frac{5 \lambda}{8 \sin (\theta)},
$$

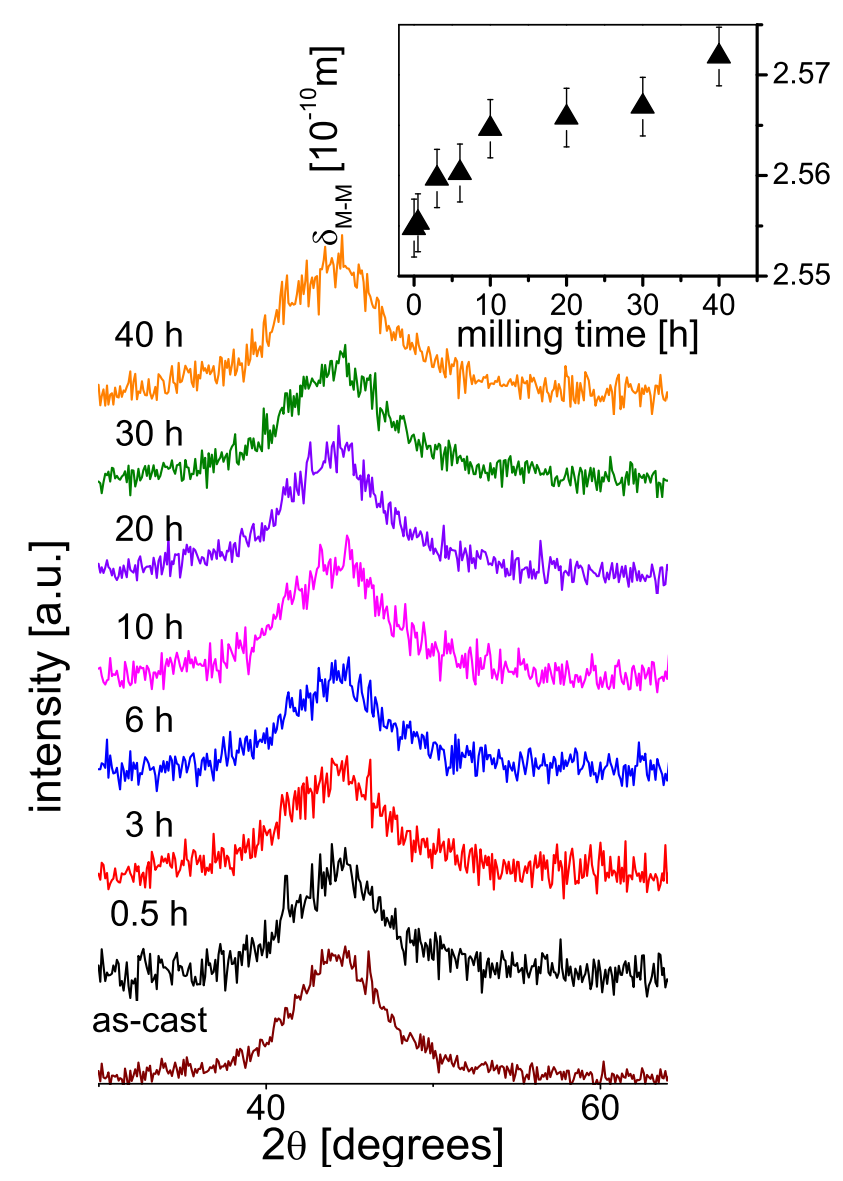

FIG. 1. XRD patterns of the as-cast ribbon and powder samples after different milling times. Inset shows the evolution of the metal-metal distance as a function of milling time. where $\lambda$ is the wavelength of the used radiation. The width of the fitted Fe-Fe distance distribution is $\sim 0.50 \mathrm{~nm}$, independently of the milling time.

Figure 2 shows the milling time dependence of room temperature values of the spontaneous magnetization and the average hyperfine magnetic field. Both parameters are well correlated and increase monotonically with the milling time. This magnetic enhancement is also reflected in the evolution of $T_{C}$. At this temperature, these samples exhibit a second order phase transition, which implies a peak in the magnetic entropy change, $\Delta S_{M}{ }^{p k}$, as shown in Figure 3. This peak value reduces after $0.5 \mathrm{~h}$ milling, although further milling leads to a monotonous increase of $\Delta S_{M}^{p k}$, reaching similar values to that of the ribbon sample after $20 \mathrm{~h}$ milling (see inset of Fig. 3). The initial reduction of $\Delta S_{M}{ }^{p k}$ after powdering the sample is not reflected in the spontaneous magnetization, which even increases from 44.8 to $51.4 \mathrm{emu} / \mathrm{g}$. However, a clear broadening of the magnetocaloric peak and a smaller slope in the $\sigma_{0}(T)$ curve of sample milled $0.5 \mathrm{~h}$ with respect to the ribbon one, should indicate a broader distribution of Curie temperatures in powder samples. The peak temperature, $T^{p k}$, which is almost coincident with $T_{C}$, at moderately small fields for these materials, ${ }^{10}$ also increases with milling from $325 \mathrm{~K}$, for melt spun ribbon, to $395 \mathrm{~K}$ after $40 \mathrm{~h}$ milling (see inset of Fig. 3). It is worth mentioning that the demagnetizing factor $N=1 / 3$ for approximately spherical powder particles should slightly modify the results shown in Figure 3, but those changes would not affect the conclusions of this work.

In order to evidence the relationship between the magnetic properties and the $\mathrm{Fe}-\mathrm{Fe}$ distance, Figure 4 shows $T_{C}$ versus the average metal-metal distance calculated from XRD data. A linear correlation is found indicating that as the $\mathrm{Fe}-\mathrm{Fe}$ distance increases, the ferromagnetic character of the powder is enhanced. This result is in agreement with literature data on pressure effect on the magnetism on Fe-based alloys without phase transformation. In those cases, the contraction of the structure due to the increase of pressure reduces both magnetization and $T_{C}{ }^{11,12}$ Moreover, considering the Slater-Pauling curve for $\mathrm{Fe}-\mathrm{Co}$, the magnetic moment of $\mathrm{Fe}$ atoms increases as the separation between atoms

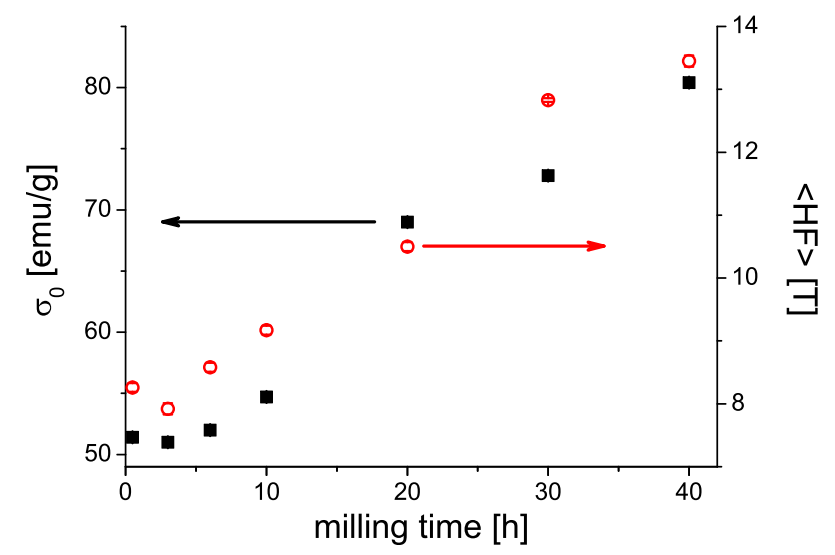

FIG. 2. Milling time dependence of the room temperature spontaneous magnetization and average hyperfine magnetic field. Error bars are smaller than the symbol sizes. 


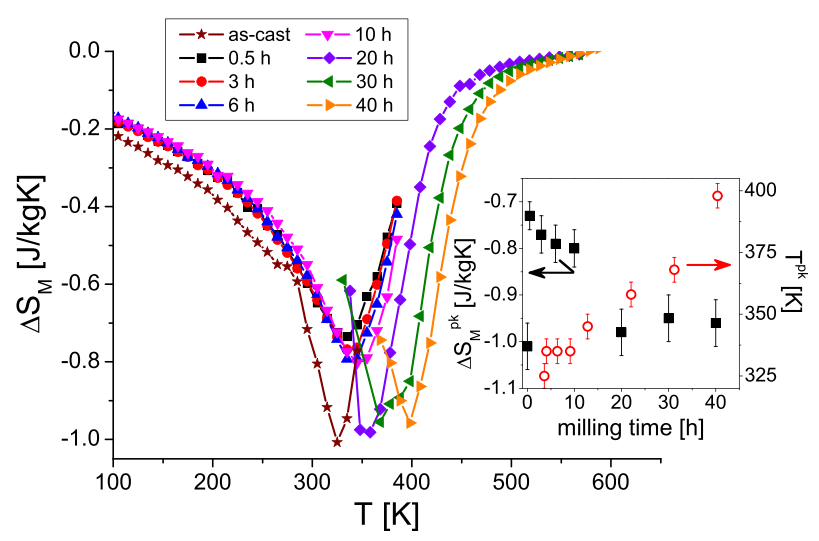

FIG. 3. Magnetic entropy change for as-cast ribbon and powder samples milled for different times. Inset shows the milling time dependence of the peak entropy change and peak temperature.

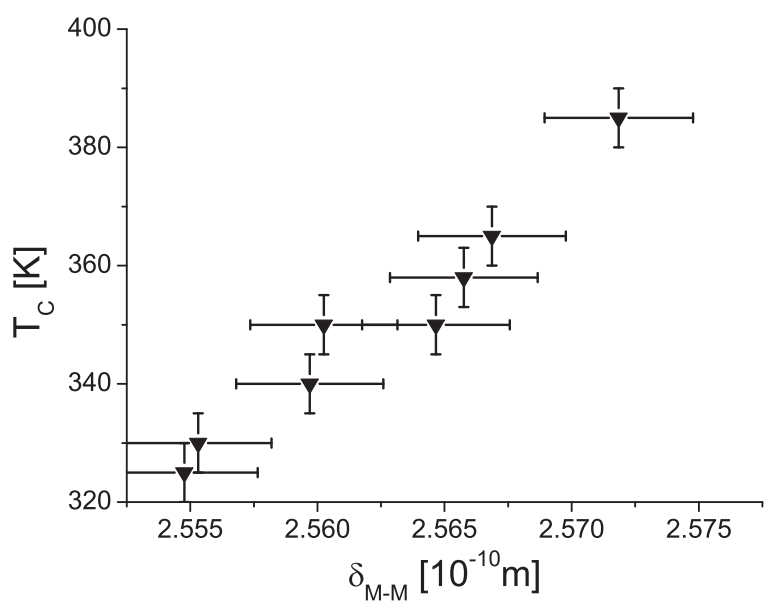

FIG. 4. Curie temperature as a function of the average metal-metal distance.

increases. ${ }^{13}$ However, it should be noted that a distribution in the $\mathrm{Fe}-\mathrm{Fe}$ distance intrinsic in the amorphous structures would produce a distribution of the exchange coupling constant, as studied by Gallagher et al. ${ }^{14}$ This also originates a broadening of the magnetocaloric peak. ${ }^{15}$ Nevertheless, even if this distribution should have an influence, as in our case the width of the $\mathrm{Fe}-\mathrm{Fe}$ distance distribution remains constant for the different samples, the differences observed in their magnetocaloric response should not be ascribed to this effect.

\section{CONCLUSIONS}

Amorphous $\mathrm{Fe}_{75} \mathrm{Nb}_{10} \mathrm{~B}_{15}$ ribbons were powdered via ball milling and the evolution of the microstructure and magnetic properties as a function of milling time were studied. From this study, some conclusions can be derived:
- The amorphous structure of the initial ribbon was retained during milling but an increase of the distance between $\mathrm{Fe}$ atoms was observed as milling progresses.

- Some changes have been observed in the magnetic properties ascribed to an increase in the $\mathrm{Fe}-\mathrm{Fe}$ distance as milling time increases: the Curie temperature of the powder samples continuously increases, the spontaneous magnetization and the average hyperfine magnetic field also increase, as well as the magnetic entropy change of the powder samples.

- The magnetic entropy change observed for short milling times is lower than those observed for the ribbon sample even though spontaneous magnetization increases after short milling. This effect is ascribed to a broader distribution of Curie temperatures in the powder samples with respect to that of the ribbon sample.

\section{ACKNOWLEDGMENTS}

This work was supported by the Spanish Ministry of Science and Innovation (MICINN) and EU FEDER (project MAT2010-20537), the PAI of the Regional Government of Andalucía (RGA) (project P10-FQM-6462), and the United States Office of Naval Research (Project N00014-11-10311). J.J.I. acknowledges a research fellowship from RGA.

${ }^{1}$ V. Franco, J. S. Blázquez, B. D. Ingale, and A. Conde, Annu. Rev. Mater. Res. 42, 305-342 (2012).

${ }^{2}$ V. K. Pecharsky and K. A. Gschneidner, Jr., Phys. Rev. Lett. 78, 4494-4497 (1997).

${ }^{3}$ F. Q. Zhao, W. Dagula, O. Tegus, T. J. Gortenmulder, E. Bruck, and K. H. J. Buschow, IEEE Trans. Mag. 41, 3754-3756 (2005).

${ }^{4}$ Y. X. Zhang, Z. G. Liu, H. H. Zhang, and X. N. Xu, Mater. Lett. 45, 91-94 (2000).

${ }^{5}$ C. Suryanarayana, Prog. Mater. Sci. 46, 1-184 (2001).

${ }^{6}$ J. J. Ipus, J. S. Blázquez, C. F. Conde, V. Franco, J. M. Borrego, S. Lozano-Perez, and A. Conde, Intermetallics 49, 98-105 (2014).

${ }^{7}$ R. A. Brand, J. Lauer, and D. M. Herlach, J. Phys. F: Met. Phys. 13, 675-683 (1983).

${ }^{8}$ See supplementary material at http://dx.doi.org/10.1063/1.4866700 for Mössbauer spectra and hyperfine field distributions for as-milled samples.

${ }^{9}$ T. Egami, Rapidly Solidified Alloys: Processes, Structures, Properties, Applications, edited by H. H. Liebermann (Marcel Dekker, Inc., New York, 1993), p. 232.

${ }^{10}$ V. Franco, A. Conde, M. D. Kuzmin, and J. M. Romero-Enrique, J. Appl. Phys. 105, 07A917 (2009).

${ }^{11}$ I. V. Medvedeva, Y. S. Bersenev, A. A. Ganin, K. Bärner, J. W. Schünemann, and K. Heinemann, J. Magn. Magn. Mater. 124, 293-297 (1993).

${ }^{12}$ A. Martínez, J. J. Romero, F. Bartolomé, L. M. García, F. Baudelet, A. Hernando, and P. Crespo, Appl. Phys. Lett. 101, 022412 (2012).

${ }^{13}$ R. M. Bozorth, Ferromagnetism (Van Nostrand, Princeton, 1968).

${ }^{14}$ K. A. Gallagher, M. A. Willard, W. N. Zabenkin, D. E. Laughlin, and M. E. McHenry, J. Appl. Phys. 85, 5130-5132 (1999).

${ }^{15}$ N. J. Jones, H. Ucar, J. J. Ipus, M. E. McHenry, and D. E. Laughlin, J. Appl. Phys. 111, 07A334 (2012). 